\title{
EFFICIENCY OF SOME INSECTICIDES ON SUGAR BEET FLY, PEGOMYIA MIXTA (VILL.) IN THE FIELD
}

\author{
SAID, A. A.A. ${ }^{1}$, F.A.H. SHAHEEN ${ }^{1}$, E.A.H. SHERIEF ${ }^{2}$ and H.A.M. FOUAD ${ }^{2}$ \\ 1 Pesticides Dept., Fac. of Agric., Mansoura Univ. \\ 2 Plant Protec. Res. Ins., ARC, Dokki-Giza, Egypt
}

(Manuscript received 26 February 2012)

\begin{abstract}
The objective of the present work was to compare the field efficiency of seven tested pesticides, Acarone 72\% EC (Profenofos), Selecron $72 \%$ EC (Profenofos), Sumithion 50\% EC (Fenitrothion), Diazonixy 60\% EC (Diazinon), Chinook 35\% SC (Imidacloprid), Vertimec $1.8 \%$ EC (Avermectin) and Actara 25\% WG (Thiamethoxam), against larvae of the sugar beet fly, Pegomyia mixta on planting sugar beet in Sharkia Governorate during 2008/2009 and 2009/2010 sugar beet growing seasons. Data obtained revealed that Acarone ( $72 \%$ EC) gave the highest initial reduction in insect population $(58.95 \%)$, followed by Diazonixy (56.68\%). Selecron ( $72 \%$ EC) was the lowest effective $(51.43 \%)$ in the first season, while in the second season, Acarone (72\% EC), Selecron (72\% EC) and Sumithion (50\% EC) achieved excellent control against $P$. mixta. Reduction percentages were 90.37, 90.13 and $88.16 \%$ after one day of spraying (initial kill).

Acarone $(72 \% \mathrm{EC})$ increased the percentage of sugar content comparing with the other treatments, whereas, there were no differences between Sumithion (50\% EC) and Control. The highest purity percentage was obtained from Selecron (72\% EC) and Sumithion (50\% $\quad$ EC) treatments $(90.76 \%$ and $89.33 \%$ respectively) in the first season, while in the second season, Acarone $(72 \% \mathrm{EC})$ and Vertimec $(1.8 \% \mathrm{EC})$ increased the percentage of sugar content comparing with the other treatments, $(19.70 \%$ and $19.40 \%$ ). While, Selecron (72\% EC) and control decreased those percentages of sugar ( $16.10 \%$ and $14.70 \%)$. Selecron ( $72 \%$ EC) and Actara (25\% WG) were increased purity percentage $(94.71 \%$ and $92.84 \%$ respectively).
\end{abstract}

Keywords: Pegomyia mixta, Pesticides, Profenofos, Fenitrothion, Diazinon, Imidacloprid, Avermectin, Thiamethoxam, Root yield and Sugar yield.

\section{INTRODUCTION}

Sugar beet (Beta vulgaris L.) is one of the most important sugar crops in the world (it produces annually about $40 \%$ of sugar production all over the world) (Anonymous, 2009). Sugar beet plants attract a considerable number of insect pests, among most important of them is the sugar beet fly, Pegomyia mixta (Diptera: Anthomyiidae). Abelentseva and Kreminskaya (1987) used Chlorophos and Phathalophos for controlling the beet leaf miner, Pegomyia betae on sugar beet. 
Phathalophos (Phosmet 20\% E C) at rate 2.5 - $5 \mathrm{Kg}$ in 100-400 litres water was more effective than Chlorophos [Trichlorfon]. Shaheen (1989) used four insecticides (Basudin, Lannate, Sumithion and Reldan) on beet fly Pegomyia mixta (Vill). All of this pesticides significantly reduced the number of Pegomyia mixta (Vill)) on sugar beet plants. Basudin was very effective than others. Bassyouny and Bleih (1996) in Egypt mentioned that Nuvacron was the most effective insecticide followed by Febronil in controlling the beet leaf miner, P. mixta. The present work was carried out to evaluate certain conventional and non-conventional insecticides against $P$. mixta infesting sugar beet plants and their effect on Juice quality, root yield and sugar yield of sugar beet at Sharkia Governorate during two successive growing seasons.

\section{MATERIALS AND METHODS}

\section{1- Experimental design:}

The experiments were carried out at Kafr El-hamam village, Zagazig district, Sharkia governorate, during the two successive seasons, 2008/2009 and 2009/2010. The experimental area was divided into plots of $6 \times 7 \mathrm{~m}\left(42 \mathrm{~m}^{2}\right)$ each arranged in a randomized complete block design with four replicates for each treatment and the untreated control. The common sugar beet variety Baraca was cultivated of Mid. of November. The normal agriculture practice was followed. The insecticides were sprayed using motor sprayer (Kubota) to give good coverage of the tested plants. Sugar beet plants were sprayed at intervals samples of five plants were collected randomly from each plot (20 plants/ treatment) just before and hence forward at $1^{\text {st }}$, $3^{\text {rd }}, 5^{\text {th }}, 7^{\text {th }}, 9^{\text {th }}$ and $11^{\text {th }}$ day from spraying. Samples were taken to laboratory for inspection.

Percentage of reduction ( $\mathrm{R} \%$ ) were calculated according to the formula of Henderson and Tilton (1955) as follows:

$$
\% \mathrm{R}=1-\left(\frac{\text { Insect No in check before spray }}{\text { Insect No in check after spray }} X \frac{\text { Insect No in treatment after spray }}{\text { Insect No in treatment before spray }}\right) X 100
$$

\section{2- Pesticides:}

Four insecticides via Acarone $72 \%$ E.C, Selecron $72 \%$ E.C, Sumithion $50 \%$ E.C and Diazonixy $60 \%$ E.C were included in the first season. While, in the second season seven insecticides namely Acarone $72 \%$ E.C, Selecron $72 \%$ E.C, Sumithion 50\% E.C, Diazonixy 60\% E.C, Chinook 35\% S.C, Vertimec 1.8\% E.C and Actara 25\% W.G were tested for control of sugar beet fly. The common name and rate of applications are used in table (1). 
Table 1. The chemicals tested and their rates in this study were as follows.

\begin{tabular}{|c|c|c|c|}
\hline No. & Trade name & Common name & $\begin{array}{l}\text { Recommended application } \\
\text { rate/100 L. of water }\end{array}$ \\
\hline 1 & Acarone $72 \%$ E.C & \multirow{2}{*}{ Profenofos } & $375 \mathrm{~cm}^{3}$ \\
\hline 2 & Selecron $72 \%$ E.C & & $375 \mathrm{~cm}^{3}$ \\
\hline 3 & Sumithion $50 \%$ E.C & Fenitrothion & $450 \mathrm{~cm}^{3}$ \\
\hline 4 & Diazonixy $60 \%$ E.C & Diazinon & $250 \mathrm{~cm}^{3}$ \\
\hline 5 & Chinook 35\% S.C & Imidacloprid & $150 \mathrm{~cm}^{3}$ \\
\hline 6 & Vertimec $1.8 \%$ E.C & avermectin & $40 \mathrm{~cm}^{3}$ \\
\hline 7 & Actara $25 \%$ W.G & Thiamethoxam & $20 \mathrm{gm}$ \\
\hline
\end{tabular}

\section{3- Statistical analysis:}

Statistical analysis were carried out to determine the differences between treatment and days after spraying by using one way analysis of variance (ANOVA) (Costat, 1990). Duncan's multiple range test (Duncan, 1955) was applied at 5\% probability level.

\section{4- Juice quality, root yield and sugar yield determinations:}

For estimating the effect of the tested insecticides on sugar beet yield. Quantity and percentage of sugar at harvest, 20 plants were taken from each treatment and the leaves were cut-off. The roots were cleaned and weighted to calculate the root yield. Sugar extractable was determined at the Department of Pesticide, Faculty of Agriculture, Mansoura University. Juice quality and sugar yield were calculated as follow:

1. Total soluble solids (TSS \%) which was determined using handle refractometer. according to Simon et al. (1980).

2. Sucrose percentage (\%) was determined in fresh roots polarmetrically using lead acetate according to the methods of Le-Docte (1927).

3. Purity percentage was calculated according to the following formula: apparent purity $\%=$ sucrose $\% /$ TSS $\%$ X 100. According to Poschenok (1976).

4. Sugar yield $($ ton/fed $)=$ root yield $X$ sugar extractable $\%$. 


\section{RESULTS AND DISCUSSION}

\section{1- Efficiency of tested pesticides against larvae of Pegomyia mixta:}

In the first season results were tabulated in Table (2) and illustrated in Fig.(1) data showed that, the activity of the tested compounds was varied according to the chemical structure of the compound, where Acarone ( $72 \% \mathrm{EC})$ gave the highest initial reduction $(58.95 \%)$, followed by Diazonixy (60\% EC) $(56.68 \%)$. Selecron (72\% EC) was the lowest effective on the population of sugar beet fly. Results also indicated that after $3,5,7,9,11$ days from spraying the reduction reached $100 \%$ except for Selecron (98.97\%) after 3days of application.

As for the residual effect, Acarone, Sumithion and Diazonixy induced the best results, recording $100 \%$ reduction followed by Selecron $(99.79 \%)$ in the first season.

Date in Table (3) and Fig.(1) indicated that Acarone, Selecron and Sumithion afforded excellent control against $P$. mixta reduction percentages were $90.37,90.13$ and $88.16 \%$ after one day of spraying (initial kill), respectively. Each of these insecticides in addition to Diazonixy, Vertimec and Chinook achieved 100\% reduction from 7days till 9days after application. Acarone, Selecron, Diazonixy, and Sumithion gave the highest efficiency in reducing sugar beet fly population with a residual effect of $100 \%$ reduction, followed by Chinook (98.99\%), Vertimec (98.48\%) and Actara $(97.13 \%)$ reduction, respectively in the second season.

These results are in agreement with data obtained by Bassyouny and Khalafalla (1996) reported that Carbosulfan was significantly effective than Profenofos against Pegomyia mixta Vill Boyd in both irrigated and non-irrigated sugar beet fields. Also, it appeared that irrigation did not significantly effect the toxicity of Carbosulfan to the considered insect. Shalaby (2001) in Egypt reported that reduction in blotches of $P$. mixta larvae were most reduced by Selecron and Marshall, Jojoba application in the third rank reducing the insect infestation by $26.53 \%$ in the first season and $33.80 \%$ in the second one. However, the poorest result was recorded for Foamier which reduced the number of $P$. mixta blotches by 7.27 and $21.44 \%$ in both seasons, respectively. Talha (2001) in Egypt evaluated some insecticides against different larval instars of $P$. mixta in sugar beet fields. The obtained results showed that Diazinoxy $60 \%$ E.C. and Diazol $60 \%$ E.C. had high activity against $P$. mixta. 
Table 2. Efficiency of some pesticides against larvae of Pegomyia mixta in sugar beet leaves during 2008/2009 at Sharkia Governorate.

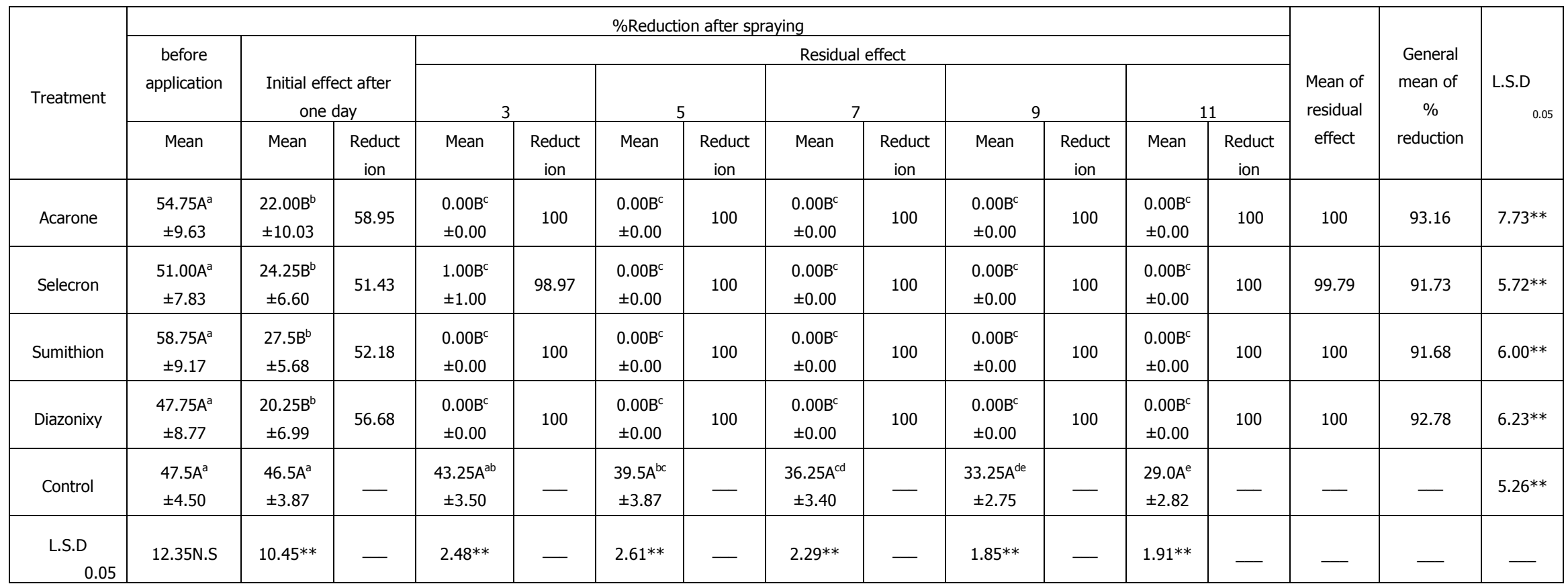

*Means followed the same capital letter in a column for different pesticides or small letter in row of each pesticides at different times are not significantly different at $5 \%$ level of Probability (Duncan's Multiple Rang Test). 
Table 3. Efficiency of some pesticides against larvae of Pegomyia mixta in sugar beet leaves during 2009/2010 at Sharkia Governorate.

\begin{tabular}{|c|c|c|c|c|c|c|c|c|c|c|c|c|c|c|c|c|}
\hline \multirow{4}{*}{ Treatment } & \multicolumn{13}{|c|}{ \%Reduction after spraying } & \multirow{4}{*}{$\begin{array}{c}\text { Mean of } \\
\text { residual } \\
\text { effect }\end{array}$} & \multirow{4}{*}{$\begin{array}{c}\text { General } \\
\text { mean of } \\
\% \\
\text { reduction }\end{array}$} & \multirow{4}{*}{$\begin{array}{l}\text { L.S.D } \\
\quad 0.05\end{array}$} \\
\hline & \multirow{3}{*}{$\begin{array}{c}\text { before } \\
\text { application } \\
\text { Mean }\end{array}$} & \multirow{2}{*}{\multicolumn{2}{|c|}{$\begin{array}{c}\text { Initial effect after } \\
\text { one day }\end{array}$}} & \multicolumn{10}{|c|}{ Residual effect } & & & \\
\hline & & & & \multicolumn{2}{|c|}{3} & \multicolumn{2}{|c|}{5} & \multicolumn{2}{|c|}{7} & \multicolumn{2}{|c|}{9} & \multicolumn{2}{|c|}{11} & & & \\
\hline & & Mean & $\begin{array}{c}\text { Reduct } \\
\text { ion }\end{array}$ & Mean & $\begin{array}{c}\text { Reduct } \\
\text { ion }\end{array}$ & Mean & $\begin{array}{c}\text { Reduct } \\
\text { ion }\end{array}$ & Mean & $\begin{array}{l}\text { Reduct } \\
\text { ion }\end{array}$ & Mean & $\begin{array}{c}\text { Reduct } \\
\text { ion }\end{array}$ & Mean & $\begin{array}{c}\text { Reduct } \\
\text { ion }\end{array}$ & & & \\
\hline Acarone & $\begin{array}{l}r r, v \circ A^{a} \\
\pm 0,07\end{array}$ & $\begin{array}{l}r, r \circ B^{b} \\
\pm r, q \wedge\end{array}$ & $q_{\cdot, r v}$ & $\begin{array}{l}0.00 C^{b} \\
\pm \cdot, \cdot\end{array}$ & $1 \ldots$ & $\begin{array}{c}0.00 \mathrm{~B}^{\mathrm{b}} \\
\pm \cdot, \cdot\end{array}$ & 100 & $\begin{array}{l}\cdot .00 \mathrm{~B}^{\mathrm{b}} \\
\pm \cdot, \cdot .\end{array}$ & 100 & $\begin{array}{l}\cdot, \cdot 0 \mathrm{~B}^{\mathrm{b}} \\
\pm \cdot, \cdot\end{array}$ & 100 & $\begin{array}{l}\cdot .00 \mathrm{C}^{\mathrm{b}} \\
\pm \cdot, \cdot,\end{array}$ & 100 & 100 & 98.39 & $3.51 * *$ \\
\hline Selecron & $\begin{array}{l}r \Lambda, \cdots A^{a} \\
\pm r, \infty 0\end{array}$ & $\begin{array}{l}r, v \circ B^{b} \\
\pm r, v_{O}\end{array}$ & $q \cdot, 14$ & $\begin{array}{l}\cdot, \cdot 0 \mathrm{C}^{\mathrm{C}} \\
\pm \cdot, .,\end{array}$ & $1 \ldots$ & $\begin{array}{l}0.00 \mathrm{~B}^{\mathrm{c}} \\
\pm \cdot, \cdot \\
\end{array}$ & 100 & $\begin{array}{l}\cdot .00 \mathrm{~B}^{\mathrm{C}} \\
\pm \cdot, \cdot .\end{array}$ & 100 & $\begin{array}{l}\cdot, \cdot \mathrm{OB}^{\mathrm{C}} \\
\pm \cdot,, .\end{array}$ & 100 & $\begin{array}{l}\cdot .00 \mathrm{C}^{\mathrm{c}} \\
\pm \cdot, \cdots\end{array}$ & 100 & 100 & 98.36 & $2.50 * *$ \\
\hline Sumithion & $\begin{array}{l}\varepsilon r, r \circ A^{a} \\
\pm Y, \wedge \varepsilon\end{array}$ & $\begin{array}{l}0.00 \mathrm{~B}^{\mathrm{b}} \\
\pm 0,9 \varepsilon\end{array}$ & $M \Lambda, 17$ & $\begin{array}{l}\cdot .00 \mathrm{C}^{\mathrm{b}} \\
\pm \cdot, \cdot,\end{array}$ & $1 \ldots$ & $\begin{array}{l}. .00 \mathrm{~B}^{\mathrm{b}} \\
\pm \cdot, \cdot\end{array}$ & 100 & $\begin{array}{l}\cdot, \cdot 0 \mathrm{~B}^{\mathrm{b}} \\
\pm \cdot, \cdot\end{array}$ & 100 & $\begin{array}{l}\cdot, \cdot 0 \mathrm{~B}^{\mathrm{b}} \\
\pm \cdot, \cdot .\end{array}$ & 100 & $\begin{array}{l}\cdot .00 \mathrm{C}^{\mathrm{b}} \\
\pm \cdot, \cdot\end{array}$ & 100 & 100 & 98.03 & $5.47^{* *}$ \\
\hline Diazonixy & $\begin{array}{l}r V, r \circ A^{a} \\
\pm \varepsilon, Y \wedge\end{array}$ & $\begin{array}{l}\varepsilon, v \circ B^{b} \\
\pm r, 0\end{array}$ & $\Delta Y, r \varepsilon$ & $\begin{array}{l}0.00 C^{c} \\
\pm \cdot, \ldots\end{array}$ & $1 \ldots$ & $\begin{array}{l}\cdot .00 \mathrm{~B}^{\mathrm{C}} \\
\pm \cdot, \cdot,\end{array}$ & 100 & $\begin{array}{l}\cdot .00 \mathrm{~B}^{\mathrm{C}} \\
\pm \cdot, \cdot .\end{array}$ & 100 & $\begin{array}{l}. .00 \mathrm{~B}^{\mathrm{C}} \\
\pm \cdot, \cdot .\end{array}$ & 100 & $\begin{array}{l}.00 \mathrm{C}^{\mathrm{c}} \\
\pm \cdot, .\end{array}$ & 100 & 100 & 97.87 & $3.00 * *$ \\
\hline Chinook & $\begin{array}{l}\varepsilon r .00 \mathrm{~A}^{\mathrm{a}} \\
\pm \varepsilon, r \varepsilon\end{array}$ & $\begin{array}{l}7.00 \mathrm{~B}^{\mathrm{b}} \\
\pm r, \leq 4\end{array}$ & $10, v 1$ & $\begin{array}{l}1,00 \mathrm{BC}^{\mathrm{C}} \\
\pm 1, V \mathrm{r}\end{array}$ & 97,74 & $\begin{array}{l}\cdot .00 \mathrm{~B}^{\mathrm{c}} \\
\pm \cdot, \cdot\end{array}$ & 100 & $\begin{array}{l}\cdot, \cdot O \mathrm{~B}^{\mathrm{C}} \\
\pm \cdot, \cdot\end{array}$ & 100 & $\begin{array}{l}\cdot .00 \mathrm{~B}^{\mathrm{C}} \\
\pm \cdot, \cdot .\end{array}$ & 100 & $\begin{array}{c}1, \cdot 0 \mathrm{BC}^{\mathrm{C}} \\
\pm 0.81\end{array}$ & 98.34 & 98.99 & 96.78 & $3.22 * *$ \\
\hline Vertimec & $\begin{array}{l}r o, r \circ A^{a} \\
\pm r, \cdot v\end{array}$ & $\begin{array}{l}\varepsilon, v \circ B^{b} \\
\pm r, \uparrow \wedge\end{array}$ & Aт,Or & $\begin{array}{l}1, r \circ B C^{b c} \\
\pm 1, r \circ\end{array}$ & 97,70 & $\begin{array}{l}\cdot, \circ 0 \mathrm{~B}^{\mathrm{bc}} \\
\pm \cdot, \text { ov }\end{array}$ & 98.73 & $\begin{array}{l}\cdot .00 \mathrm{~B}^{\mathrm{c}} \\
\pm \cdot, \cdot .\end{array}$ & 100 & $\begin{array}{l}\cdot .00 \mathrm{~B}^{\mathrm{C}} \\
\pm \cdot, \cdot .\end{array}$ & $1 \ldots$ & $\begin{array}{l}1, \circ \mathrm{BC}^{\mathrm{bc}} \\
\pm 1.00\end{array}$ & 97.03 & 98.48 & 96.43 & $4.06 * *$ \\
\hline Actara & $\begin{array}{l}\varepsilon \cdot, \vee \circ A^{a} \\
\pm \wedge, 0\end{array}$ & $\begin{array}{l}\Upsilon, 0 \cdot B^{b} \\
\pm \varepsilon, V Y\end{array}$ & $\wedge \varepsilon, \cdot \varepsilon$ & $\begin{array}{l}r .00 \mathrm{~B}^{\mathrm{b}} \\
\pm r, \varepsilon \varepsilon\end{array}$ & $9 \Gamma, .0$ & $\begin{array}{l}\cdot, v \circ \mathrm{B}^{\mathrm{b}} \\
\pm \cdot, 9 \circ\end{array}$ & 98.35 & $\begin{array}{l}\cdot, 00 \mathrm{~B}^{\mathrm{b}} \\
\pm 1, \ldots\end{array}$ & $9 \wedge, 9 \wedge$ & $\begin{array}{l}\cdot r \circ B^{b} \\
\pm 0.50\end{array}$ & 99.55 & $\begin{array}{l}r, \circ 0 B^{b} \\
\pm 1, r q\end{array}$ & 95.72 & 97.13 & 94.95 & $5.68 * *$ \\
\hline Control & $\begin{array}{l}\varepsilon 1, Y \circ A^{C} \\
\pm r, A Y\end{array}$ & $\begin{array}{l}\varepsilon I, Y \circ A^{C} \\
\pm r, A Y\end{array}$ & - & $\begin{array}{l}\varepsilon r, \vee \circ A^{c} \\
\pm r, r\end{array}$ & - & $\begin{array}{l}\sum \tau, r \circ A^{b c} \\
\pm r, \circ q\end{array}$ & - & $\begin{array}{l}0.0 \mathrm{~A}^{\mathrm{b}} \\
\pm \varepsilon, 0 \varepsilon\end{array}$ & - & $\begin{array}{l}07, r \circ A^{a} \\
\pm \varepsilon, \wedge 0\end{array}$ & - & $\begin{array}{l}\circ 9, Y \circ A^{a} \\
\pm r, 7 r\end{array}$ & - & - & - & $5.32 * *$ \\
\hline $\begin{array}{c}\text { L.S.D } \\
0.05\end{array}$ & A,rVN.S & 0,or** & - & $r$, rq** & - & $1.94 * *$ & - & $2.40 * *$ & 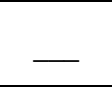 & $2.52 * *$ & - & $1.65^{* *}$ & - & - & - & - \\
\hline
\end{tabular}

*Means followed the same capital letter in a column for different pesticides or small letter in row of each pesticides at different times are not significantly different at $5 \%$ level of probability (Duncan's Multiple Rang Test). 


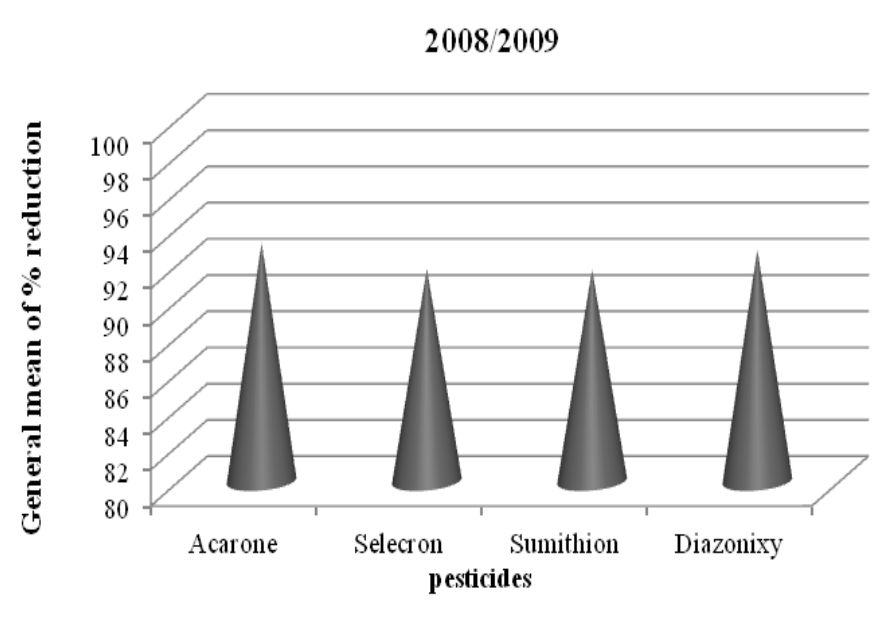

2009/2010

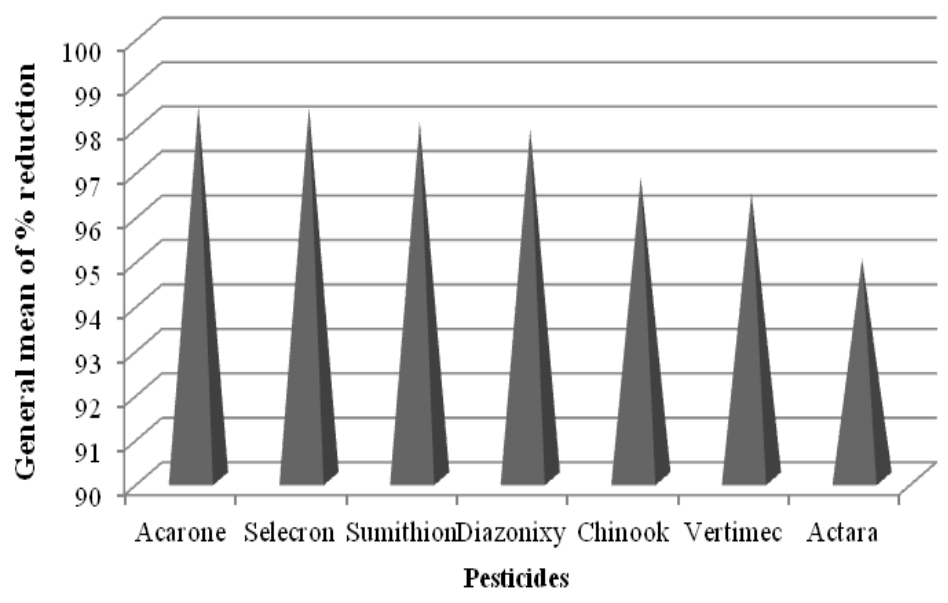

Fig. 1. General mean of reduction of beet fly, Pegomyia mixta in sugar beet leaves during 2008/2009 and 2009/2010 seasons.

\section{2- Efficiency of insecticides on sugar content, root yield and sugar yield:}

\subsection{Season 2008/2009:-}

Data in table (4) showed that Acarone increased the percentage of sugar content comparing with other treatments, whereas, there were no differences between Sumithion and Control, as well as total soluble solids (TSS \%) as shown in fig. (2). The highest purity percentage was obtained from Selecron and Sumithion $(90.76 \%$ and $89.33 \%$ respectively). On the other hand Diazonixy and control were the lowest one in juice purity percentage ( $87.03 \%$ and $87.24 \%$ respectively).

Data also indicated that Acarone and Diazonixy increased the sugar yield and roots yield (Ton / fed.) comparing with other treatments. 


\subsection{Season 2009/2010:-}

Data in table (4) indicated that Acarone and Vertimec increased the percentage of sugar content comparing with other treatments (19.70\% and $19.40 \%$ respectively). While, Selecron and control decreased those percentages of sugar (16.10\% and $14.70 \%$ respectively), as well as total soluble solids (TSS \%) as shown in fig. (2). Selecron and Actara were increased purity percentage (94.71 \% and $92.84 \%$ respectively). On the other hand, Acarone and Vertimec were the lowest one in juice purity percentage ( $85.65 \%$ and $88.18 \%$ respectively).

Data also showed that Acarone and Vertimec increased the sugar yield and roots yield (Ton / fed.) comparing with other treatments.

Table 4. Efficiency of certain insecticides on Juice quality, Root yield and Sugar yield infested by Pegomyia mixta during 2008/2009 and 2009/2010 seasons.

\begin{tabular}{|c|c|c|c|c|c|c|c|}
\hline Treatments & Season & $\begin{array}{l}\text { Root weight } \\
(\mathrm{kg}) / 20 \text { plants }\end{array}$ & $\begin{array}{c}\text { Sucrose } \\
\text { (\%) }\end{array}$ & $\begin{array}{l}\text { TSS } \\
(\%)\end{array}$ & $\begin{array}{l}\text { Purity } \\
(\%)\end{array}$ & $\begin{array}{l}\text { Roots yield } \\
\text { Ton/Fed }\end{array}$ & $\begin{array}{c}\text { Sugar } \\
\text { yield } \\
\text { Ton/Fed }\end{array}$ \\
\hline \multirow{2}{*}{ Acarone } & $2008 / 2009$ & 29.27 & 17.13 & 20.00 & 85.75 & 40.978 & 7.02 \\
\hline & $2009 / 2010$ & 29.45 & 19.70 & 23.00 & 85.65 & 41.230 & 8.12 \\
\hline \multirow{2}{*}{ Selecron } & $2008 / 2009$ & 23.00 & 14.75 & 16.25 & 90.76 & 32.200 & 4.75 \\
\hline & $2009 / 2010$ & 20.50 & 16.10 & 17.00 & 94.71 & 28.700 & 4.62 \\
\hline \multirow{2}{*}{ Sumithion } & $2008 / 2009$ & 21.58 & 13.40 & 15.00 & 89.33 & 30.212 & 4.05 \\
\hline & $2009 / 2010$ & 23.35 & 17.03 & 18.75 & 90.83 & 32.690 & 5.56 \\
\hline \multirow{2}{*}{ Diazonixy } & $2008 / 2009$ & 26.71 & 16.10 & 18.50 & 87.03 & 37.394 & 6.02 \\
\hline & $2009 / 2010$ & 25.80 & 19.10 & 21.50 & 88.84 & 36.120 & 6.89 \\
\hline Chinook & $2009 / 2010$ & 25.15 & 18.80 & 21.00 & 89.52 & 35.210 & 6.62 \\
\hline Vertimec & $2009 / 2010$ & 27.65 & 19.40 & 22.00 & 88.18 & 38.696 & 7.50 \\
\hline Actara & $2009 / 2010$ & 24.50 & 18.80 & 20.25 & 92.84 & 34.300 & 6.44 \\
\hline \multirow{2}{*}{ Control } & $2008 / 2009$ & 20.90 & 12.65 & $1 \leq .0$. & 87.24 & 29.260 & 3.70 \\
\hline & $2009 / 2010$ & 19.15 & 14.70 & 14.70 & 100 & 26.810 & 3.94 \\
\hline
\end{tabular}

TSS $=$ Total soluble solids . 


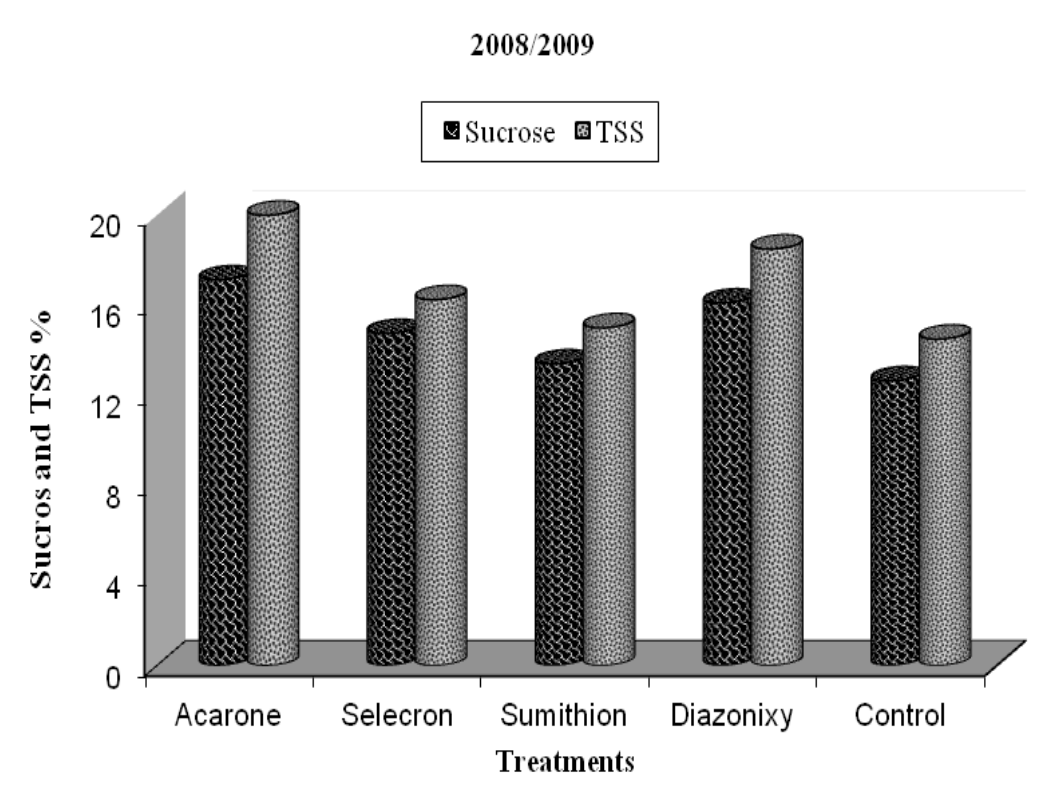

$2009 / 2010$

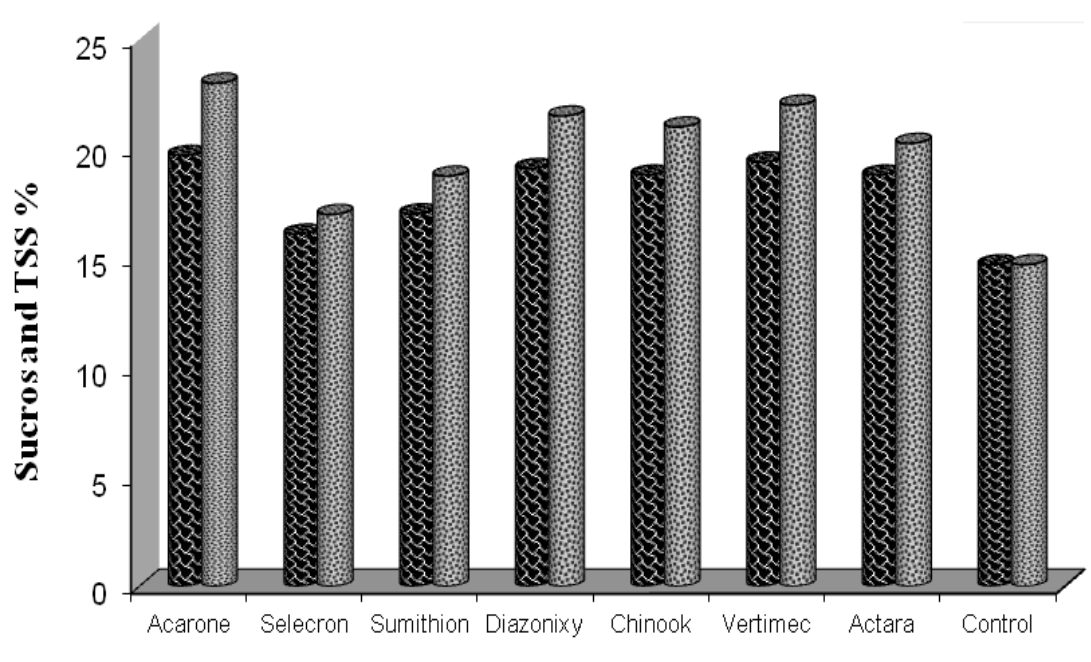

Treatments

Fig. 2. Effect of the tested insecticides on percentage of sugar and total soluble solids during 2008/2009 and 2009/2010 seasons. 


\section{REFERENCES}

1. Abelentseva, G.M and T.I. Kreminskaya. 1987. Phathalophos. Zashchita-Rastenii - Moskva. 1986. No. 11. 54.

2. Anonymous. 2009. Sugar crops council, ministry of agriculture and land reclamation.

3. Bassyouny, A. M. and E.M.E. Khalafalla. 1996. fluctuations of certain insect pests on three sugar beet varieties and their chemical control. Alex. Sci. Excha. 17(4): 381-393.

4. Bassyouny, A. M. and S. B. Bleih. 1996. Sowing dates, seasonal fluctuations and chemical control against the main insects attacking sugar beet. Alex. Sci. Exch. 17 (3): 283 - 296.

5. Costat Statistical Software. 1990. Microcomputer program analysis version 4.20, cohort software, Berkeley, CA.

6. Duncan, D.B. 1955. Multiple range and multiple F-tests. Biometrics. 11: $1-42$.

7. Henderson, C. F. and E.W. Tilton. 1955. Test with acaricides against the brown wheat mite. J. Econ. Entomol. 84: 157-161.

8. Le-Docte, A. 1927. Commercial determination of sugar beet root using the Sachr Le-Docte processes. Int. Sugar J., 29: 488 - 492. (C.F. Sugar beet nutrition, Applied Sciences Publishers LTD, London, A.P. Draycott).

9. Poschenok. 1976. Biochemical Analysis of Fruit and Vegetable Products. TataMc graw-Hill publishing company limited, New Delhi pp, $634-650$.

10. Shaheen, F.A.H. 1989. The effect of mixing Certain Pesticides with some foliar fertilizers on their stability and efficiency against some sugar beet insects. J. Agric. Sci. Mansoura Univ. 17(12): 3911-3918.

11. Shalaby, G.A.M. 2001. Ecological studies on some important sugarbeet pests and natural enemies and their control. Ph. D. Thesis, Fac. of Agric, Kafr El-Sheikh, Tanta Univ., 141 pp.

12. Simon, P.W., C.E. Peterson and R.C. Lindsay. 1980. Correlations between sensory and objective parameters of college flavor, J. Agric. Food chem. 28: $559-562$.

13. Talha, E. A. M. M. 2001. Integrated pest management of sugar-beet insects. M. Sc. Thesis, Fac. Agric, Mansoura Univ., 102 pp. 


\section{كفاءة بعض المبيدات الحشرية على ذبابة بنجر السكر فى الحقل (Pegomyia Mixta)}

على على عبدالهادى سعيد '، فؤاد عبدالله حسام الدين شاهين '، السيد على حسن

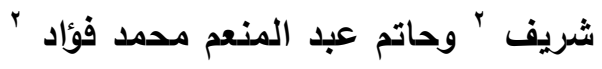

$$
\begin{aligned}
& \text { ا ـ قسم المبيد/ت- كلية الزراعة- جامعة المنصورة. }
\end{aligned}
$$

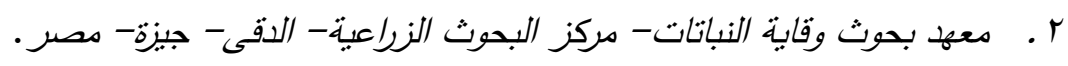

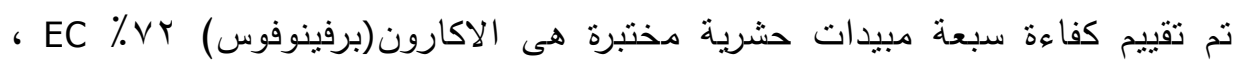

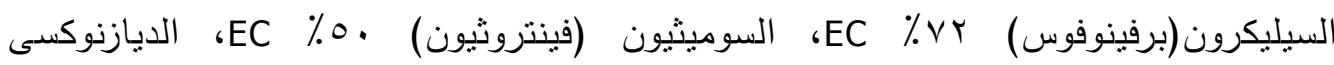

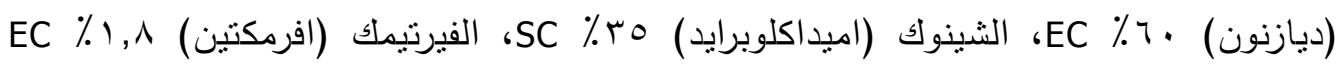

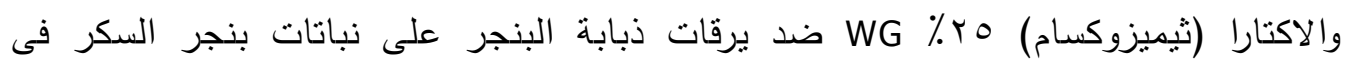

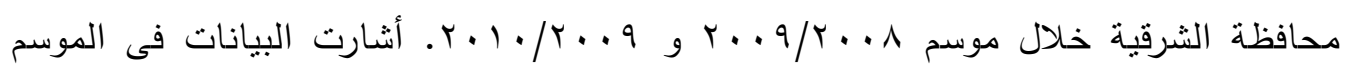

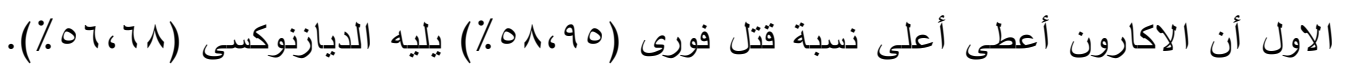

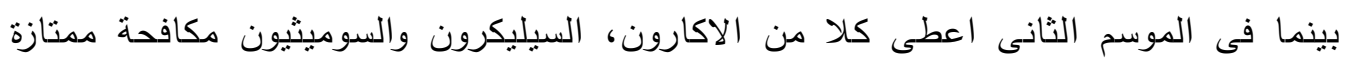

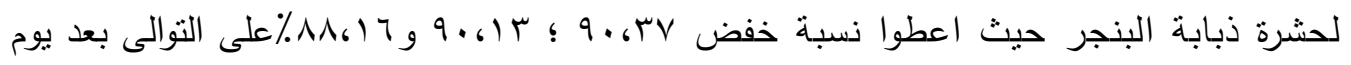
واحد من الرش (القتل الفورى). كذلك تمت دراسة تاثير هذة المبيدات على نسبة السكر والمواد الصلبة الذائبة حيث تبين فى الموسم الاول ان مركب الاكارون ادى الى زيادة ذسبة السكر

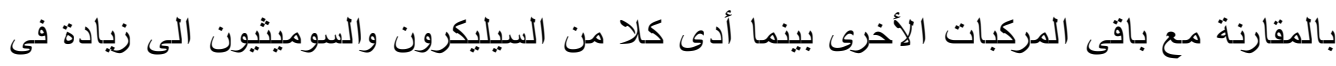

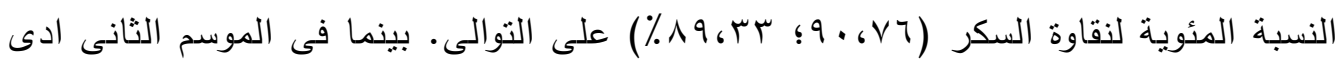

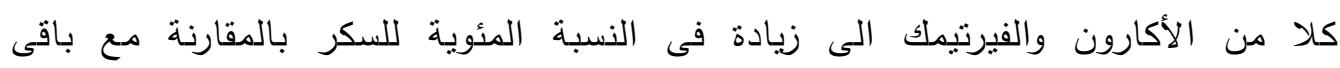

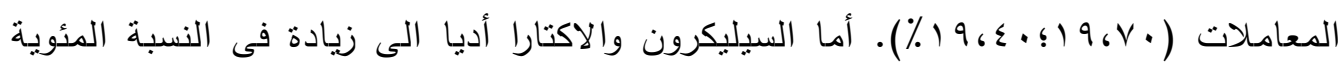

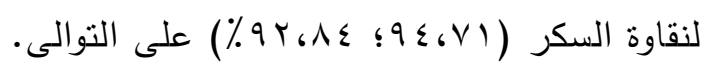

